\title{
Introduction to the special issue on CSP technologies in artificial intelligence
}

\author{
Éric Grégoire • Bertrand Mazure
}

Published online: 10 April 2015

(C) Springer Science+Business Media New York 2015

\section{Introduction}

Although constraint programming has evolved as an independent domain of research per se, it remains at the same time one of the most fertile and successful areas of artificial intelligence (A.I.). This special issue is intended to illustrate various current topics and research trends in constraint programming that pertain to A.I. It gathers a series of research contributions that range from the study of tractable classes of non-binary CSPs, to efficient algorithms for the extraction of minimal unsatisfiable subsets in constraint networks, and include the application of constraint programming for deciding the robustness of wireless sensor networks and interval methods for numerical constraint programming.

The papers in this issue are all revised and expanded research contributions based on results that were presented at the SAT and CSP technologies track at IEEE ICTAI 2013. They were selected as the papers with the highest review scores amongst the 47 full papers submitted to the track, after all the papers submitted to the track had been thoroughly reviewed by at least three reviewers. The authors of the selected papers in the track were invited to submit substantially expanded and revised contributions for this issue. Each of these expanded contributions underwent two additional reviews.

We gratefully acknowledge the reviewers for providing deep insights and useful suggestions that helped improve the papers. Finally, we would like to thank Gilles Pesant and Michela Milano, Editors in chief of Constraints, for inviting us to edit this special issue and for their expert help at the different stages of the process.

É. Grégoire $(\varangle) \cdot$ B. Mazure

CRIL, Université d'Artois, CNRS, rue Jean Souvraz SP18, 62307 Lens, France

e-mail: gregoire@cril.fr

B. Mazure

e-mail: mazure@ cril.fr 


\section{Content of this issue}

The first paper is authored by Achref El Mouelhi, Philippe Jégou and Cyril Terrioux and investigates tractables classes of CSPs. The paper introduces a way to extend the tractable BTP (Broken Triangle Property) class of binary constraints to constraints of arbitrary arities, while keeping tractability. It then studies the relation of the new class called D(ual) BTP with other tractable fragments and investigates DBTP from a practical point of view: the authors show that DBTP contains some well-known benchmarks, which provides an explanation of the efficiency of state-of-the-art solvers on them.

The second paper is by Éric Grégoire, Bertrand Mazure and Jean-Marie Lagniez: it focuses on the extraction of minimal subsets of unsatisfiable constraints (MUCs) in discrete constraint networks. This issue is central for explaining and fixing over-constrained networks. The authors question the traditional two-steps so-called destructive processes to extract one MUC, which first require a good-quality preliminary over-approximation of a MUC. They question the necessity of the first step and, step by step, build an approach that skips it and proves more efficient most often.

The third research contribution is by Luis Quesada, Lanny Sitanayah, Kenneth N. Brown, Barry O'Sullivan and Cormac J. Sreenan. The paper is concerned with the robustness of the topology of wireless sensor networks that are subject to node failures. The authors show that checking whether a network enjoys the so-called strongly $k$-robustness property is an NP-complete problem. They then develop a constraint programming approach for deciding strongly $k$-robustness and show that it outperforms a mixed-integer programming model on large problems.

The fourth paper is by Bertrand Neveu, Gilles Trombettoni and Ignacio Araya. This study is about numerical CSPs and interval methods. The main practical contribution of this paper is to show that an adaptive version of the CID (Constructive Interval Disjunction) operator can be efficient for both real-valued satisfaction and optimization problems, while needing no additional parameter value from the user.

Éric Grégoire \& Bertrand Mazure, February 2015. 